\title{
Adding Radiocarbon to the State Factor Matrix
}

\begin{abstract}
SUSAN TRUMBORE ${ }^{1}$
${ }^{1}$ Max-Planck Institute for Biogeochemistry, Jena, Germany, trumbore@bgc-jena.mpg.de

The amount of organic matter stored in litter and soil is a function of the soil forming (edaphic) factors, including climate, organisms, parent material and time. Providing quantiative understanding of each of these controls involves the selection of field sites where one of these factors can be isolated. For example, chronosequences can be used to correlate mineral stabilization mechanisms with the amount of stored carbon. The development of field locations to study such gradients is a difficult and time-consuming endeavor, so they become valuable proving grounds for a range of methods to study soil biogeochemistry. The addition of radiocarbon to studies of soil organic matter brings new information about the timescales of organic matter cycling. In addition, repeated measures of radiocarbon over time can track decadal cycling of carbon by following the incorporation and persistence of 'bomb' $14 \mathrm{C}$ produced by weapsons testing in the 1960s. This talk will update studies done with Oliver Chadwick using radiocarbon measurements to study climate and mineral controls on timescales of soil carbon cycling.
\end{abstract}

